Special issue of the 2nd International Conference on Computational and Experimental Science and Engineering (ICCESEN 2015)

\title{
Palm Vein Authentication and Verification System
}

\author{
M. CÖMERT AND A. Yildiz* \\ Mersin University, Department of Electrical and Electronics Engineering, Mersin, Turkey
}

\begin{abstract}
In this work, both hardware and software of a palm vein capture system have been designed and a prototype is produced. By using this system, a genuine database was created with age and gender information of volunteered test subjects. Widely used pre-processing, acquisition of feature vectors, and classification methods were tested on the database and results are compared with speed versus accuracy. All the modules used in hardware and software were determined as open source. Python has been chosen as a software module programming on a single board computer with Linux kernel. As a result, an appropriately running system has been built with both secure software and hardware interfaces having maximum accuracy at $95.33 \%$ while specificity is at $100 \%$.
\end{abstract}

DOI: 10.12693/APhysPolA.130.175

PACS/topics: 07.05.Pj, 42.30.Sy, 42.30.Tz

\section{Introduction}

Palm vein images are widely acceptable as a model which may give appropriate patterns for biometric classification in literature. Palm vein structures are preferable in terms of uniqueness, which covers studies about methods of imaging, pre-processing procedures, methods of acquisition feature vectors and classification methods as a biometric specification. Those studies go through with one or many of priorities which are accuracy, processing speed, portability, and security.

Veins under the human skin cannot be properly distinguished by applying the visible spectrum of light. Hemoglobin absorbs almost all the spectrum span from 760 to $1100 \mathrm{~nm}$ wavelength [1]. High contrast in the captured images was obtained between veins and their surrounding at $850 \mathrm{~nm} \mathrm{[2].} \mathrm{Therefore,} \mathrm{sensor} \mathrm{of} \mathrm{a} \mathrm{digi-}$ tal camera which is sensitive to the infrared is utilized to capture the palm vein pattern. Reflection method was preferred for palm illumination due to ease of use and simplicity of the system. The veins of the palm $3 \mathrm{~mm}$ deep under the skin can be seen in the image by this method [1].

The pre-processing method is focused to calculate image features without creating any structure of the veins [3], because, more than $95 \%$ accuracy was achieved in a previous study [4] which used matching algorithms with the same method.

There are many methods to match feature vectors of patterns [5-7]. Although accuracy is mostly dependent to feature vector subsets, usually one of the maximum similarity or minimum distance methods is chosen for matching features since equal error rate is as small as zero for these methods $[5,6]$.

\section{Hardware}

Reflection method has been implemented in visualization hardware by using 4 LEDs which deliver $1 \mathrm{~W}$

\footnotetext{
${ }^{*}$ corresponding author; e-mail: yildiz@mersin.edu.tr
}

total power ranging from 835 to $875 \mathrm{~nm}$ wavelengths with $110^{\circ}$ irradiation angle [8].

A CCD sensor with a compatible connection to embedded hardware has been selected without built-in infrared cut filter. It could be focused to $10 \pm 2 \mathrm{~cm}$ without attaching any external lens. As seen in Fig. 1, a platform has been designed to focus the sensor to the region of interest which was chosen as center of palm since a lot of veins can be captured as classification pattern [9].
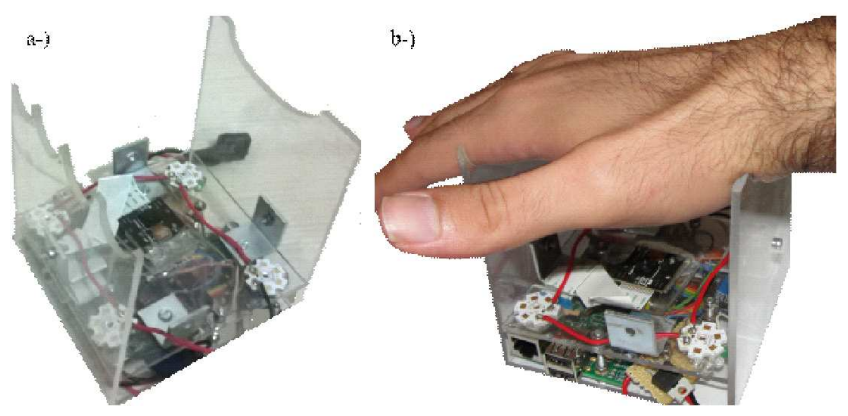

Fig. 1. (a),(b) Prototype of the hardware.

Biometric sensors should not be directly connected to a computer on any biometric classification system in order to protect personal biometric information against any digital attack. For that reason, pre-processing and acquisition feature vectors must be computed on an embedded hardware before sending the information to the computer. In this work, Raspberry Pi type B embedded platform has been chosen for both hardware modularity and application programming interfaces [10].

\section{Software}

Raspbian operating system has been chosen to run the main client software. It has been written in Python 2.7 with Picamera, Numpy and Scikit image modules preinstalled [11, 12].

Properties of camera hardware have been adjusted by means of Picamera module for hardware resolution to 


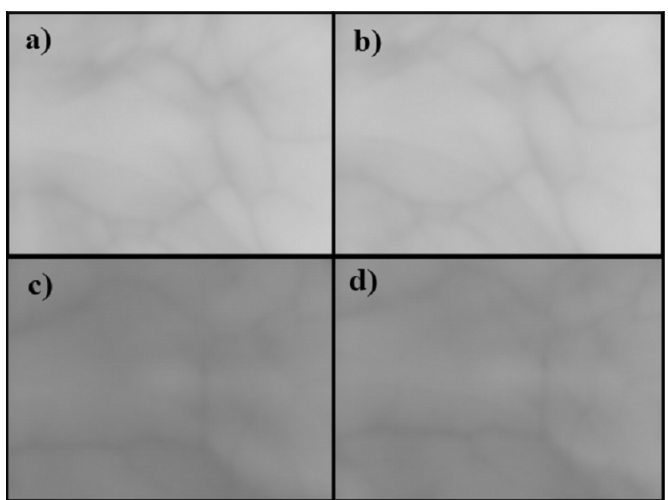

Fig. 2. Captured palm vein raw images before any preprocessing. Two images were taken at different times from (a),(b) the same male volunteer, (c),(d) the same female volunteer.

$320 \times 240$ pixels. Auto white balance support is shut down to get raw image data which is more convenient for image processing. Raw images can be seen in Fig. 2 .

Both captured image and processed image have been kept on RAM as volatile variables until process ends in order to avoid external attacks.
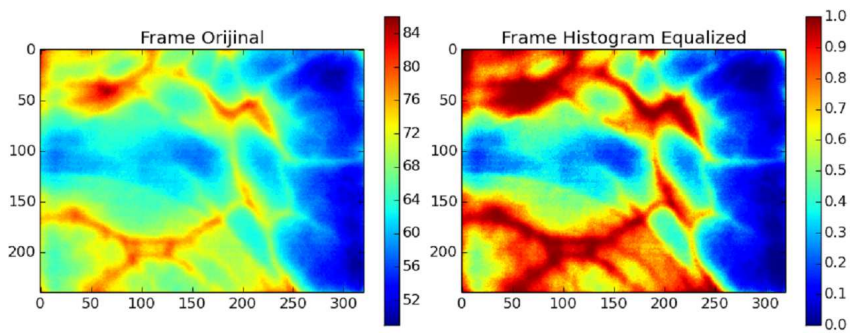

Fig. 3. (a) Before and (b) after the histogram equalization.

Basic histogram equalization [13] which normalize pixel values according to their probability distribution function, has been applied to improve the contrast in palm vein images (see Fig. 3).

The small changes in the image capturing angle could not be eliminated completely. Also, homogeneity of illumination distribution on the palm could not be accomplished by the hardware. Therefore, unwanted brightness differences on the image were inevitable. To fix this undesirable brightness, contrast limited adaptive histogram equalization (CLAHE) [14] has been applied to the image (see Fig. 4).

Local binary patterns are powerful descriptors for each pixel of the image [15]. They can be implemented with rotation and grayscale invariance up to feature set. In this study, rotation invariant variance measures of the local contrast are used as descriptor of local binaries. Variance measures are not grayscale invariant. Maximum accuracies were calculated for each of the following cases: radius of 3 pixels for $32 \times 32$ resolution, 4 pixels for $64 \times 64$ resolution, and 8 pixels for $128 \times 128$ resolutions of palm vein captured images.
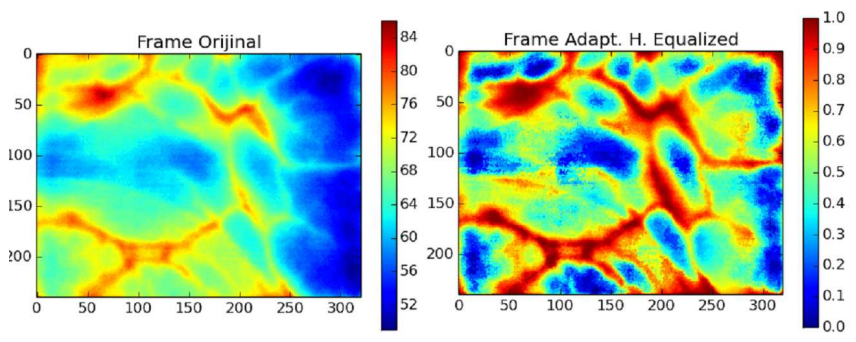

Fig. 4. Database structure.

In order to increase the accuracy, histogram of oriented gradients were used as another descriptor which achieves grayscale invariant features while both scale and rotation are fixed [16]. Maximum accuracies were calculated again for the same cases mentioned above with sub-blocks areas: $4 \times 4$ pixel-area for $32 \times 32$ resolution, $8 \times 8$ pixel-area for $64 \times 64$ resolution and $18 \times 18$ pixel-area for $128 \times 128$ resolution palm vein captured images.

In decision function, cosine similarity of feature vectors was chosen for identifying a person. Cosine similarity of the local binary patterns and histogram of oriented gradients have been calculated separately. Fusion process has been established in order to check if both local matches give the same result or not.

\section{Performance}

3 right palm vein images of 100 randomly chosen people has been taken and grouped as in Table I for examining.

TABLE I

Database structure.

\begin{tabular}{c|c}
\hline \hline Identified & Test \\
\hline 2 frames & 1 frame from 75 identified people \\
from 75 people & +3 frames from 25 unidentified people
\end{tabular}

Receiver operating characteristic (ROC) has been calculated separately for both local thresholds (see Table II). Then, fusion match results has been checked for resolution and pre-processing. The highest accuracy has been reached at $64 \times 64$ resolution (see Table II). Although higher resolutions increase true positive rates, on the other hand, they decrease specifity due to the noise.

TABLE II

Accuracy, sensitivity and specifity vs. resolution.

\begin{tabular}{c|c|c|c|c|c}
\hline \hline Res. & Acc. & Sens. & Spec. & \multicolumn{2}{|c}{$\begin{array}{c}\text { Thresholds } \\
\text { LBP } / \text { HOG }\end{array}$} \\
\hline $24 \times 24$ & 0.880 & 0.880 & 0.880 & 0.06 & 0.38 \\
$32 \times 32$ & 0.920 & 0.866 & 0.973 & 0.06 & 0.29 \\
$64 \times 64$ & 0.953 & 0.906 & 1.000 & 0.12 & 0.16 \\
$128 \times 128$ & 0.920 & 0.906 & 0.933 & 0.12 & 0.09
\end{tabular}

Image capturing process has been optimally limited to $500 \mathrm{~ms}$ to have both better quality image and real-time 
process. Pre-processing and acquisition feature methods also cost about $400 \mathrm{~ms}$ with transmission duration (UART speed is chosen as $115200 \mathrm{bit} / \mathrm{s}$ ) from embedded platform to the computer. On the user interface, matching a vein pattern of a person among 150 feature vectors take only $8.45 \mathrm{~ms}$ on Core i7 4702MQ @ $2.2 \mathrm{GHz}$ 8GB RAM. Hence, our classification and authentication prototype can be classed as a real-time system since the total process time takes less than $1 \mathrm{~s}$.

TABLE III

Power consumption of the system.

\begin{tabular}{c|c|c|c}
\hline \hline Hardware & $\begin{array}{c}\text { Current } \\
\text { consumption }[\mathrm{A}]\end{array}$ & $\begin{array}{c}\text { Voltage } \\
{[\mathrm{V}]}\end{array}$ & $\begin{array}{c}\text { Power } \\
{[\mathrm{W}]}\end{array}$ \\
\hline embedded system & 0.700 & 5.0 & 3.500 \\
camera & internal & internal & internal \\
infrared light source & 0.200 & 10.8 & 2.160 \\
USB to UART module & 0.025 & 5.0 & 0.125 \\
LED indicators & 0.060 & 3.3 & 0.198 \\
total & - & - & 5.983
\end{tabular}

\section{Conclusion and future work}

In this study, an entire palm vein recognition system was designed with both hardware and software components as a whole running system in real-time. Local binary patterns and histogram of orientated features' similarities are combined for decision function to increase the accuracy of the results. Accuracy gets higher with higher image resolution and reaches to a maximum value of $95.33 \%$ while specifity is at $100 \%$ (see Table II) with $64 \times 64$ pixels resolution. However, if resolution is increased even higher, print of the palm mixes into the vein pattern as a noise. In this case, region of interest can be shifted as a result of small position change of the hand. Additionally, the system is running in real-time since total time required is less than $1 \mathrm{~s}$. As a result, our prototype device is well-suited for $64 \times 64$ pixel resolution for person classification and authentication from palm vein image in real time.

To improve the hardware of the system, polarization filter would be used to remove palm prints in the images. In addition, diffusion paper would be used to have homogeneous light distribution all over the palm. On the software side, number of database entry would be extended to get a better matching performance.

\section{References}

[1] J.M. Cross, C.L. Smith, in: Security Technology, 1995. Proc. 29th Int. Carnahan Conf. on, Sanderstead, IEEE, 1995, p. 20.

[2] O. Nikisins, M. Greitans, R. Fuksis, M. Pudzs, Z. Serzane, BIOSIG 164, 133 (2010).

[3] Y. Zhou, A. Kumar, Inform. Forens. Secur. IEEE Trans. Biom. Compend. 6, 1259 (2011).

[4] A. Gupta, A. Malage, D. More, P. Hemane, P. Bhautmage, D. Dhandekar, in: Advances in Engineering and Technology Research (ICAETR), Proc. Int. Conf. on, Unnao, IEEE, 2014, p. 1.

[5] L. Wang, G. Leedham, D.S.Y. Cho, Pattern Recogn. 3, 920 (2008).

[6] M. Manocha, P. Kaur, Int. J. Innovat. Technol. Explor. Eng. 3, 2278 (2013).

[7] D.Y. Perwira, B.W.T. Agung, M.D. Sulistiyo, in: $I C-$ ITSI 2014, Int. Conf. on Information Technology Systems and Innovation, Bandung, 2014, p. 99.

[8] LedEngin LZ1, 2015, Oct..

[9] Raspberry Pi NoIR Camera - Infrared Camera, 2015, Oct.

[10] Raspberry Pi 1 Model B, 2015, Oct.

[11] S. van der Walt, J.L. Schönberger, J. Nunez-Iglesias, F. Boulogne, J.D. Warner, N. Yager, E. Gouillart, T. Yu, PeerJ 2, e453 (2014).

[12] S. van der Walt, S.C. Colbert, G. Varoquaux, Comput. Sci. Eng. 13, 22 (2011).

[13] P. Ladoux, C. Rosenberger, B. Dorizzi, Adv. Biometr. 5558, 1290 (2009).

[14] A. Kumar, K.V. Prathyusha, IEEE Trans. Image Process. 18, 2127 (2009).

[15] L. Mirmohamadsadeghi, A. Drygajlo, in: Biometrics (IJCB), 2011 Int. Joint Conf. on Biometrics Compendium, IEEE, Washington, DC 2011, p. 1.

[16] W. Jia, R. Hu, Y. Lei, Y. Zhao, J. Gui, Biometr. Compend. IEEE 44, 385 (2013).

[17] M. Mohan, J. Saxena, K. Teckchandani, P. Pandey, in: IWOBI 2015, 4th Int. Work Conf. on Bioinspired Intelligence, San Sebastian, IEEE, 2015, p. 151. 